\title{
Clinical Effect and Safety Evaluation of Paroxetine and Sertraline Hydrochloride Used for Depression Treatment after Cerebral Stroke
}

\author{
Fu Beibei, Meng Shuqing* \\ Department of Neurology, Renmin Hospital, Hubei University of Medicine, Shiyan Renmin Hospital, Shiyan, \\ Hubei Province, China,442000
}

Address: Chaoyang District Road No. 39, Shiyan City, Hubei Prov, P.R. China, 442000

*Corresponding author: Meng Shuqing, E-mail: hubeiwenbo@163.com

Keywords: paroxetine; sertraline hydrochloride; cerebral stroke; depression; curative effect; safety

\begin{abstract}
To analyze clinical effect and safety of paroxetine and sertraline hydrochloride used for depression treatment after cerebral stroke, 58 patients with depression after cerebral stroke were chosen as the objects of study, and they were divided into two groups at random according to the numeration table. 29 patients in the control group were treated with paroxetine, while the 29 patients in the experimental group were treated with sertraline hydrochloride. Clinical effects and safety of both groups were compared and summarized. Results show that, before treatment, Self-rating Anxiety Scale (SAS) score and Self-rating Depression Scale (SDS) score of both groups had no statistical significance ( $>0.05$ ). After the treatment for $2 \mathrm{w}, 5 \mathrm{w}$ and $7 \mathrm{w}$, negative mood improved significantly, and score difference of both groups at two points in time after the treatment had no statistical significance ( $>>0.05$ ). But according to adverse effects of both groups, the total occurrence rate of adverse effects in experimental group was obviously lower than that of the control group, and the difference had statistical significance $(\mathrm{P}<0.05)$. Thus, it can be concluded that both paroxetine and sertraline hydrochloride can gain the ideal effect when being used to treat depression after cerebral stroke, and they contribute to improving patients' negative mood. But by contrast, adverse effects of sertraline hydrochloride are fewer, which indicates its safety is higher and deserves to be promoted and applied clinically.
\end{abstract}

\section{INTRODUCTION}

Cerebral stroke is common clinically, with high disability rate, and it results in severe impacts on patients' physical and psychological health, and living quality. Depression is a common complication of patients after cerebral stroke. The clinical survey data show that among all cerebral stroke patients, occurrence rate of depression is as high as $40 \%-50 \%$. The main clinical manifestations include abnormal low mood, few words, sleep disorders and interest decline, etc. Some even have the idea of suicide. These cause severe impacts on clinical treatment and prognosis $[1,2]$. Thus, during clinical treatment of cerebral stroke patients, protopathy requires to be treated. Meanwhile, collaborative treatment of depression should be stressed for the patients in need so as to maximize curative effect. Paroxetine and sertraline hydrochloride are common medicines of depression after cerebral stroke, but the clinical reports on treatment value of both are few. 58 patients with depression after cerebral stroke were chosen in this paper to compare and analyze treatment effect and safety of the two medicines so as to provide reference for clinical medication.

\section{DATA AND METHOD}

\subsection{General data}

The 58 patients with depression after cerebral stroke were chosen from the hospital from April 2015 to December 2017. All of them received relevant examinations and were diagnosed with depression after cerebral stroke. They conformed to relevant diagnosis standards set in cerebrovascular disease academic conference and depression complied with relevant diagnosis 
standards in Chinese Classification and Diagnosis of Mental Diseases. The research content was approved by relevant ethics committee. Besides, patients and their family members knew the situation and voluntarily signed relevant letter of consent. The following patients were excluded: accompanied with other organ function diseases, with severe mental disorders, with tumor and with hyperalgesia to the medicines used in the research; those who did not the research; those who could not complete the research due to other factors.

Among the 58 patients, there were 33 male patients and 25 female patients, with the age of 36-75 and average age of $59.35 \pm 6.77$. The course of disease was $1-10 \mathrm{~m}$, and the average course of disease is $(5.35 \pm 1.41) \mathrm{m}$. there were 14 patients with combined cerebral infarction, 10 patients with coronary heart disease, 28 patients with hypertension and 6 patients with cerebral hemorrhage. All patients were divided into control group (29 patients) and experimental group (29 patients) at random according to the numeration table. Baseline information difference of both groups had no statistical significance $(\mathrm{P}>0.05)$.

\subsection{Method}

The patients in both groups received symptomatic treatment and rehabilitation treatment of cerebrovascular disease. On this basis, the experimental group took orally sertraline hydrochloride (manufacturer: Pfizer; approval No.: GYZZ H10980141) in the morning. The starting dosage was adjusted to $50.0 \mathrm{mg} /$ time (once per day), and the dosage was increased to $100 \mathrm{mg} /$ time according to patients' clinical effect. The control group took orally paroxetine (manufacturer: Tianjin Smith Kline \& French laboratories Ltd.; approval No.: GYZZ H10950043) after breakfast, 20.0mg/time, once per day. Both groups were treated for $8 \mathrm{w}$.

\subsection{Curative effect criterion}

(1) The negative mood was assessed before the treatment as well as $3 \mathrm{w}, 5 \mathrm{w}$ and $7 \mathrm{w}$ after the treatment. SAS was used to score, and the total score of the scale is 80 . The higher score means more severe anxiety. The total score of SDS is 80, and the higher score means more severe depression [3,4]. (2) Occurrence rates of adverse effects were observed and recorded after clinical treatment.

\subsection{Statistical method}

All data generated in the research were processed with statistics software package (SPSS21.0). Independent sample value test was used for measurement data "mean \pm standard deviation". Chi-square value test was used for enumeration data "rate". If the difference has statistical significance, it is expressed with $\mathrm{P}<0.05$.

\section{RESULTS}

\subsection{SAS and SDS score difference of both groups at each pint in time}

Before treatment, SAS and SDS score difference of both groups had no statistical significance ( $\mathrm{p}>0.05$ ). After treatment for 3w, 5w and 7w, negative mood improved significantly, and SAS and SDS score difference of both groups after treatment had no statistical significance ( $>0.05)$ (Tab.1).

Tab.1 SAS and SDS score difference of both groups at each pint in time $(\bar{x} \pm s)$

\begin{tabular}{cccc}
\hline Group & Time & SAS (score) & SDS (score) \\
\hline & Before treatment & $53.52 \pm 4.79$ & $51.03 \pm 5.03$ \\
Experimental group(29) & Treatment for 3w & $32.35 \pm 4.62^{*}$ & $29.79 \pm 5.00^{*}$ \\
& Treatment for 5w & $23.77 \pm 4.03^{*}$ & $22.23 \pm 4.44^{*}$ \\
& Treatment for 7w & $14.53 \pm 3.77^{*}$ & $12.01 \pm 3.82^{*}$ \\
& Before treatment & $52.79 \pm 4.82$ & $50.97 \pm 5.00$ \\
& Treatment for 3w & $33.39 \pm 4.71^{*}$ & $31.13 \pm 4.98^{*}$ \\
Control group(29) & Treatment for 5w & $25.92 \pm 4.00^{*}$ & $25.00 \pm 4.76^{*}$ \\
& Treatment for 7w & $16.66 \pm 3.45^{*}$ & $14.43 \pm 3.93^{*}$ \\
\hline
\end{tabular}

Note: (1) compared with pre-treatment, * means $\mathrm{P}<0.05$; $t$ values in the experimental group are $17.13,25.59$ and 34.45 respectively; $t$ values in the control group are 15.50, 23.10 and 32.82 
respectively; (2) in the inter-group comparison at each point in time after treatment, $\mathrm{P}>0.05$; $t$ value is 1.18 after the treatment for $3 \mathrm{w}$; $t$ value is 2.04 after the treatment for $5 \mathrm{w}$; $t$ value is 2.24 after the treatment for $7 \mathrm{w}$;

\subsection{Comparison of occurrence rate of adverse effects after treatment}

Seeing from adverse effects of both groups, total occurrence rate of adverse effects in the experimental group was significantly lower than that in the control group, and the difference had statistical significance $(\mathrm{P}<0.05)$ (Tab.2).

Tab.2 Comparison of occurrence rate of adverse effects after treatment $\quad(n, \%)$

\begin{tabular}{|c|c|c|c|c|c|c|}
\hline Group & $\begin{array}{l}\mathrm{N} \\
\mathrm{o} .\end{array}$ & $\begin{array}{l}\text { Mouth parched and tongue } \\
\text { scorched }\end{array}$ & $\begin{array}{l}\text { Nausea and } \\
\text { vomiting }\end{array}$ & $\begin{array}{l}\text { Headac } \\
\text { he }\end{array}$ & $\begin{array}{l}\text { Nocturnal } \\
\text { enuresis }\end{array}$ & $\begin{array}{c}\text { Tota } \\
1\end{array}$ \\
\hline $\begin{array}{l}\text { Experimental } \\
\text { group }\end{array}$ & 29 & 1 & 1 & 0 & 1 & $\begin{array}{c}10.3 \\
4\end{array}$ \\
\hline Control group & 29 & 2 & 2 & 2 & 1 & $\begin{array}{c}24.1 \\
4\end{array}$ \\
\hline$\chi^{2}$ & l & l & l & / & l & 5.74 \\
\hline $\mathrm{P}$ & l & l & / & l & l & $\begin{array}{c}<0.0 \\
5\end{array}$ \\
\hline
\end{tabular}

\section{DISCUSSIONS}

Depression is a common and severe complication for the patients with cerebral stroke, and causes the threat to patients' physical and psychological health, with the different degree of depression. In current stage, clinical treatment of depression after cerebral stroke is mainly achieved with non-medicine scheme. The common treatment means include rehabilitation therapy, electric convulsion therapy, psychotherapy and hyperbaric oxygen therapy, etc. Drug therapy is a common and non-physical therapy. In the early stage, tricyclic/ tetracyclic antidepressant drugs are often used, but traditional drugs have negative reactions with different degrees. So, their use is hindered in clinical promotion [5,6]. 5-HT selective seratonin re-uptake inhibitors (SSRIs), selective noradrenalin reuptake inhibitors (NARIs), and selective dopamine reuptake inhibitors (NDRIs) belong to new clinical antidepressant drugs, and they gain more ideal clinical effect because of their lower adverse effects. In this research, paroxetine and sertraline hydrochloride are typical selective 5-HT re-uptake inhibitors which achieve the treatment through promoting the increase of synaptic cleft 5-HT concentration [7,8].

Based on the research results, SAS and SDS scoring indexes show that both groups have no significant differences before the treatment and at each point in time after the treatment. This indicates that the application effect of the two medicines is ideal. After the treatment, SAS and SDS scores are obviously lower than that before the treatment. The data indicate that the two medicines can gain good clinical effect and contribute to improving patients' anxiety, depression and other negative mood as well as their clinical manifestations depression. In addition, in terms of adverse effects after the treatment, the occurrence rate of experimental group is significantly lower than that of control group, which indicates that compared with sertraline hydrochloride, paroxetine contributes better to reducing adverse effects. The reason may be that after sertraline is taken, its affinity to $\alpha, \beta, \mathrm{D}, 5$-HT and acetyl choline receptors is lower $[9,10]$. The patients with depression after cerebral stroke are mostly middle-aged and old patients. Considering such patients are mostly accompanied with other types of underlying diseases, sertraline hydrochloride is recommended clinically.

In conclusion, both paroxetine and sertraline hydrochloride can gain the ideal effect and contribute to improving patients; negative mood when they are used to treat the patients with depression after cerebral stroke. But by contrast, sertraline hydrochloride leads to fewer adverse effects, indicating its safety is higher and deserves to be promoted and applied clinically. 


\section{REFERENCES}

[1] Khasanova DR, Zhitkova IuV, Safiullina AA, Efficacy of adepress (paroxetine) in the treatment of depression in patients with chronic cerebral ischemia in the late recovery phase of ischemic stroke with the estimation of the velocity of passive transmembrane ion transport, $\mathrm{Zh}$ Nevrol Psikhiatr Im S S Korsakova. 2012;112(3 Pt 2):27-30.

[2] Horváth S, Karányi Z, Harcos P, Clinical effectiveness and safety of paroxetine in post-stroke depression: results from a phase 4, open label, multicenter clinical trial with 26 weeks of follow-up,Orv Hetil. 2006 Dec 17;147(50):2397-404. Hungarian.

[3] Paolucci S, Antonucci G, Grasso MG,Post-stroke depression, antidepressant treatment and rehabilitation results. A case-control study,Cerebrovasc Dis. 2001;12(3):264-71.

[4] Erfurth A1, Loew M, Wendler G,Depressive disorders in neurologic rehabilitation: therapy with paroxetine, Psychiatr Prax. 2001 Jan;28(1):43-4.

[5] Sun Y, Liang Y, Jiao Y, Lin J, Qu H, Xu J, Zhao C, Comparative efficacy and acceptability of antidepressant treatment in poststroke depression: a multiple-treatments meta-analysis,BMJ Open. 2017 Aug 3;7(8): e016499.

[6] Karaiskos D, Tzavellas E, Spengos K, Vassilopoulou S, Paparrigopoulos T,Duloxetine versus citalopram and sertraline in the treatment of poststroke depression, anxiety, and fatigue, $\mathrm{J}$ Neuropsychiatry Clin Neurosci. 2012 Summer;24(3):349-53.

[7] Peng L, Zhang X, Kang DY, Liu XT, Hong Q,Effectiveness and safety of Wuling capsule for post stroke depression: a systematic review,Complement Ther Med. 2014 Jun;22(3):549-66.

[8] Nie RR, Huang CH,Post-stroke depression treated with acupuncture and moxibustion: an evaluation of therapeutic effect and safety,.Zhongguo Zhen Jiu. 2013 Jun;33(6):490-4. Chinese.

[9] Ramasubbu R,Minor strokes related to paroxetine discontinuation in an elderly subject: emergent adverse events,Can J Psychiatry. 2003 May;48(4):281-2.

[10] Chen JW, Xie SQ,Agomelatine versus paroxetine in treating depressive and anxiety symptoms in patients with chronic kidney disease,Neuropsychiatr Dis Treat. 2018 Feb 16; 14:547-552. 